\title{
The Numerically Optimized Regulator and the Functional Renormalization Group
}

I. G. Márián ${ }^{a}$, U. D. Jentschura ${ }^{b, c}$ and I. Nándoric,d

a) University of Debrecen, P.O. Box 105, H-4010 Debrecen, Hungary

b) Missouri University of Science and Technology, Rolla, Missouri 65409-0640, USA

c) MTA-DE Particle Physics Research Group, P.O. Box 51, H-4001 Debrecen, Hungary

d) MTA Atomki, P.O. Box 51, H-4001 Debrecen, Hungary

We aim to optimize the functional form of the compactly supported smooth (CSS) regulator within the functional renormalization group (RG), in the framework of bosonized two-dimensional Quantum Electrodynamics $\left(\mathrm{QED}_{2}\right)$ and of the three-dimensional $O(N=1)$ scalar field theory in the local potential approximation (LPA). The principle of minimal sensitivity (PMS) is used for the optimization of the CSS regulator, recovering all the major types of regulators in appropriate limits. Within the investigated class of functional forms, a thorough investigation of the CSS regulator, optimized with two different normalizations within the PMS method, confirms that the functional form of a regulator first proposed by Litim is optimal within the LPA. However, Litim's exact form leads to a kink in the regulator function. A form of the CSS regulator, numerically close to Litim's limit while maintaining infinite differentiability, remains compatible with the gradient expansion to all orders. A smooth analytic behaviour of the regulator is ensured by a small, but finite value of the exponential fall-off parameter in the CSS regulator. Consequently, a compactly supported regulator, in a parameter regime close to Litim's optimized form, but regularized with an exponential factor, appears to have favorable properties and could be used to address the scheme dependence of the functional renormalization group, at least within the the approximations employed in the studies reported here.

\section{Contents}

1 Introduction

2 Regulators and Models 23

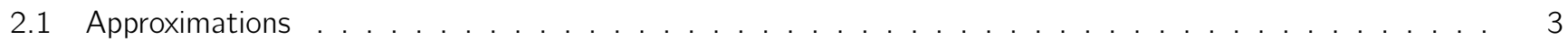

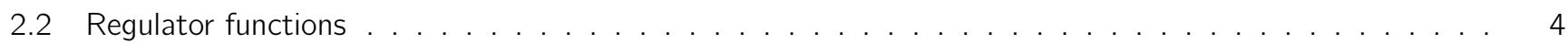

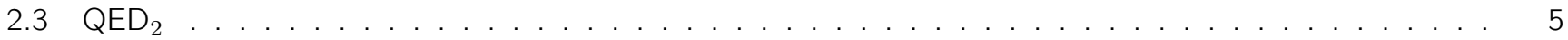

3 Results obtained by the RG study of QED $_{2} \quad 6$

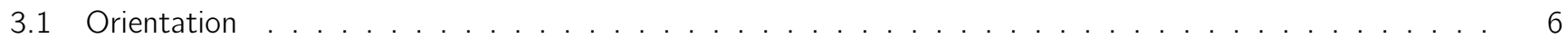

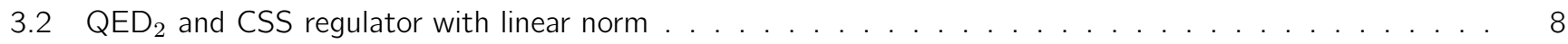

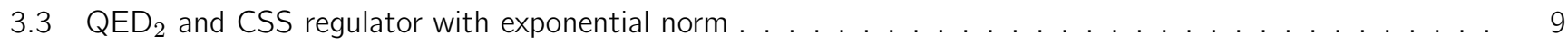

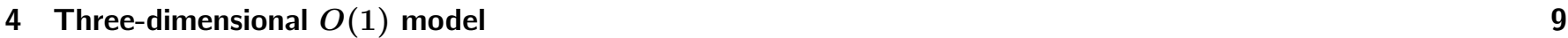

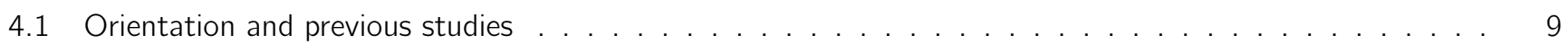

4.2 Three-dimensional $\boldsymbol{O}(\mathbf{1})$ model and CSS regulator with exponential norm . . . . . . . . . . . . 11

5 Summary 


\section{Introduction}

In particle physics, theories and models are defined at high energies, where symmetry considerations are valid, but the measurements are performed at relatively low energies. The low-energy behaviour of field-theoretical models is determined by the renormalization group. Usually, perturbative renormalization is sufficient. However, there are special situations, for example the confinement of quarks into hadrons, where non-perturbative treatments are needed. Renormalization can be performed non-perturbatively by means of the functional renormalization group ( $R G)$ method $[1,2,3,4]$ which was applied successfully in many cases. The following functional RG equation for scalar fields [2] (Wetterich equation)

$$
k \partial_{k} \Gamma_{k}[\varphi]=\frac{1}{2} \operatorname{Tr}\left[\left(k \partial_{k} R_{k}\right) /\left(\Gamma_{k}^{(2)}[\varphi]+R_{k}\right)\right]
$$

may be derived for the blocked effective action $\Gamma_{k}$ which interpolates between the bare action $\Gamma_{k \rightarrow \Lambda} \equiv S$ and the full quantum effective action $\Gamma_{k \rightarrow 0} \equiv \Gamma$ where $k$ is the running momentum scale. The second functional derivative of the blocked action is represented by $\Gamma_{k}^{(2)}$, and the trace $\operatorname{Tr}$ stands for the momentum integration. $R_{k}$ is the regulator function which is assumed to fulfill the conditions $R_{k}(p \rightarrow 0)>0$, as well as $R_{k \rightarrow 0}(p)=0$ and $R_{k \rightarrow \Lambda}(p)=\infty$. The physical results obtained by the exact RG equation are independent of the particular choice of the regulator [5].

The RG equation constitutes a functional partial differential equation and it is therefore not possible to indicate general solutions. Hence, approximations are required. One of the commonly used systematic approximations is the truncated gradient expansion [6]. The necessity of approximations implies that the RG flow depends on the choice of the regulator function, i.e., on the renormalization scheme ("scheme dependence"). In principle, therefore, physical results could become scheme-dependent. The comparison of results obtained using various types of regulator functions (see Refs. [7, 9, 8, 11, $10,14,6,18,12,13,16,17,19])$ is indicated. In order to increase the predicting power of the RG method, an optimization of the scheme-dependence is required. A rather general optimization procedure (Litim-Pawlowski method [7, 8]) leads to Litim's regulator [7]. In typical cases, it agrees well with experimental data [9], and furthermore, the corresponding RG equation can be mapped onto the Polchinski RG at least in the leading order of the gradient expansion [10]. Its disadvantage is that it is non-differentiable and thus incompatible with the gradient expansion [10,11]. Another optimization scenario is based on the principle of minimal sensitivity (PMS, see Ref. [12]), where the optimal parameters of a given regulator are chosen such as to make the physical quantities as insensitive as possible to any conceivable changes of the parameters entering the regulator. Its advantage is that it can be used at any order of the gradient expansion, its disadvantage is that regulators of different functional form cannot easily be compared to each other based on the PMS alone. The "optimization" then proceeds under the implicit assumption that the PMS method converges to the optimized values rather than a non-optimal "saddle point" in the parameter space. In the following, we reproduce a number of known results for optimum parameters in limiting cases, and we reassure ourselves that in all cases considered, the results of the PMS method are consistent with the globally optimal values of the parameters that define the regulator within the functional forms discussed in the current paper.

Recently, a new type of regulator function, the compactly supported smooth (CSS) regulator [13], has been introduced which encompasses all major types of regulator functions discussed so far in the literature, in appropriate limits. Thus, it can be used to compare various regulator functions to each other in the framework of the PMS optimization method. Moreover, it is a smooth, infinitely differentiable function, and it has a compact support (it is non-zero only in a finite range). Therefore, it can be applied to consider the "Litim limit" at any order of the gradient expansion.

The purpose of this paper is to study the scheme-dependence of the functional RG in the framework of the bosonized $\mathrm{QED}_{2}$ (Ref. [14]) and the $O(N=1$ ) symmetric scalar field theory [9], within the local potential approximation (which is the leading order of the gradient expansion). We use the CSS regulator function [13]. Our goal is (i) to optimize the parameters of the CSS regulator in LPA using the PMS optimization procedure, and (ii) to compare the results obtained by the PMS strategy to the one advocated by Litim. For this, the CSS regulator has been investigated with two types of normalization.

The organization of this paper is as follows. In Sec. 2, wegive a brief overview of the regulators and field-theoretical models studied here. In particular, we consider typical approximations used in solving RG equations and major types of regulator functions. Furthermore, known results on QED $_{2}$ are summarized. In Secs. 3 and 4, the optimization of the CSS regulator 
is presented in the framework of $\mathrm{QED}_{2}$ and the $O(N=1)$ model, respectively. Conclusions are reserved for Sec. 5.

\section{Regulators and Models}

\subsection{Approximations}

In order to solve the RG equation (1), one of the commonly used systematic approximations is the truncated gradient (i.e., derivative) expansion where $\Gamma_{k}$ is expanded in powers of the derivative of the field,

$$
\Gamma_{k}[\varphi]=\int \mathrm{d}^{d} x\left[V_{k}(\varphi)+Z_{k}(\varphi) \frac{1}{2}\left(\partial_{\mu} \varphi\right)^{2}+\ldots\right] .
$$

In the local potential approximation (LPA), higher derivative terms are neglected and the wavefunction renormalization is set equal to a constant, i.e., $Z_{k} \equiv 1$.

The solution of the RG equations sometimes requires further approximations, e.g., the Taylor or Fourier series of the potential $V_{k}(\varphi)$ in terms of the field variable (with a truncation $N_{\text {cut }}$ )

$$
\begin{aligned}
V_{k}(\varphi) & =\sum_{n=1}^{N_{\text {cut }}} \frac{g_{n}(k)}{n !} \varphi^{n}, \\
V_{k}(\varphi) & =\sum_{n=1}^{N_{\text {cut }}} u_{n}(k) \cos (n \beta \varphi),
\end{aligned}
$$

where the scale-dependence is encoded in the coupling constants $g_{n}(k)$ or $u_{n}(k)$.

A third systematic possibility to expand the RG flow is provided by the so-called amplitude expansion which is frequently used. Let us write the RG equation obtained in LPA in the following form,

$$
\begin{aligned}
k \partial_{k} V_{k} & =-\alpha_{d} k^{d} \int_{0}^{\infty} \mathrm{d} y \frac{d r}{d y} \frac{y^{d / 2+1}}{P^{2}+\omega}, \\
r(y) & =\frac{R_{k}(p)}{p^{2}}, \quad y=\frac{p^{2}}{k^{2}}, \quad P^{2}=[1+r(y)] y, \quad \omega=\frac{V_{k}^{\prime \prime}}{k^{2}} .
\end{aligned}
$$

The $d$-dimensional solid angle reads as $\Omega_{d}=2 \pi^{d / 2} / \Gamma(d / 2)$, while $\alpha_{d}=\Omega_{d} /\left(2(2 \pi)^{d}\right)$ and $r(y)$ is the dimensionless regulator function. The amplitude expansion reads as

$$
\begin{aligned}
k \partial_{k} V_{k} & =\sum_{m=1}^{\infty} \frac{2 m}{d} a_{2 m-d}(-\omega)^{m-1} \\
a_{n} & =\int_{0}^{\infty} \mathrm{d} y\left(-\frac{d}{2} \frac{r^{\prime}}{(1+r)^{d / 2+1}}\right) P^{-n} .
\end{aligned}
$$

When using the Litim-Pawlowski optimization $[7,8]$, the optimal choice for the parameters of the regulator functions can be determined in such a way to provides us the most favorable convergence of the amplitude expansion [7, 9]. Among the regulators, Litim's optimized one [7] is seen to lead to the fastest convergence of the amplitude expansion. Here and in the following sections of this article, the "optimum" parameters are always to be understood in terms of the additional approximations employed in the optimization process, e.g., the LPA. The caveat is that parameters which are determined as optimal within a specific, leading-order approximation to the RG flow, are implicitly assumed to approximate the optimum parameters within different and more detailed approximation schemes. Without this assumption, or a variation of this assumption, the determination of "optimum" parameters within any approximation to the RG flow would not be meaningful. We adopt the implicit assumption and proceed accordingly. The most general definition of an "optimized RG flow" would otherwise encompass the "shortest" RG trajectory in theory space, where "short" trajectories are quantified 
in terms of criteria for the gap in the flow equation. This sense of optimization is independent of any approximation scheme, see Ref. [8]. In the simple case of a derivative expansion to lowest order, this boils down to an optimization of the regulator function which is the subject of the current work.

\subsection{Regulator functions}

Various choices for regulator functions have already been discussed in the literature in a great detail. For example, one of the most frequently used regulator functions is the exponential one [2]

$$
r_{\exp }(y)=\frac{a}{\exp \left(c_{2} y^{b}\right)-1}
$$

with $b \geq 1$. Within the LPA, a favorable choice for the parameters has been determined as $a=1, c_{2}=\ln (2)$ and $b=1.44$, based on the Litim-Pawlowski method [7, 8]. Another example is the power-law type regulator [3]

$$
r_{\text {pow }}(y)=\frac{a}{y^{b}},
$$

with $a=1$ and $b=2$ as optimal choices. Finally, one of the most popular regulators is the Litim choice [7],

$$
r_{\mathrm{opt}}^{\mathrm{gen}}(y)=a\left(\frac{1}{y^{b}}-1\right) \Theta(1-y)
$$

which is a continuous (but not differentiable) function with compact support [the Heaviside step function is denoted as $\Theta(y)$ ]. The parameters $b=1$ and $a=1$ are obtained as a result of the Litim-Pawlowski optimization method in LPA.

The recently introduced CSS regulator [13] is defined as

$$
r_{\mathrm{css}}^{\mathrm{gen}}(y)=\frac{\exp \left[c y_{0}^{b} /\left(f-h y_{0}^{b}\right)\right]-1}{\exp \left[c y^{b} /\left(f-h y^{b}\right)\right]-1} \Theta\left(f-h y^{b}\right) .
$$

It is infinitely differentiable at $f-h y^{b}=0$ in view of the essential singularity of the exponential function at infinity. Let us note, that the number of free parameters in (10) can be reduced by setting $f=1$ without loss of generality,

$$
r_{\mathrm{css}}^{\operatorname{modif}}(y)=\frac{\exp \left[c y_{0}^{b} /\left(1-h y_{0}^{b}\right)\right]-1}{\exp \left[c y^{b} /\left(1-h y^{b}\right)\right]-1} \Theta\left(1-h y^{b}\right) .
$$

Before we go on to discuss the properties of the smooth regulators, we should remark that that a similar smoothing problem in nuclear physics has already been solved by introducing the so-called Salamon-Vertse potential which can be related to the CSS regulator [15]. It is easy to show that both forms (10) and (11) of the CSS regulator have the property to recover all major types of regulators: Litim's optimized (9), the power-law (8) and the exponential (7) ones,

$$
\begin{aligned}
\lim _{c \rightarrow 0, f=h=1} r_{\mathrm{css}}^{\mathrm{gen}} & =\lim _{c \rightarrow 0, h=1} r_{\mathrm{css}}^{\text {modif }}=\frac{y_{0}^{b}\left(y^{-b}-1\right)}{1-y_{0}^{b}} \Theta(1-y), \\
\lim _{f \rightarrow \infty} r_{\mathrm{css}}^{\mathrm{gen}} & =\lim _{h \rightarrow 0, c \rightarrow 0} r_{\mathrm{css}}^{\text {modif }}=\frac{y_{0}^{b}}{y^{b}}, \\
\lim _{h \rightarrow 0, c=f} r_{\mathrm{css}}^{\mathrm{gen}}(y) & =\lim _{h \rightarrow 0, c \rightarrow 1} r_{\mathrm{css}}^{\operatorname{modif}}=\frac{\exp \left[y_{0}^{b}\right]-1}{\exp \left[y^{b}\right]-1} .
\end{aligned}
$$

In order to further reduce the number of free parameters of the CSS regulator, $y_{0}$ can be chosen so that the numerator of (11) becomes a linear function of the parameter $c$

$$
r_{\mathrm{css}}^{\mathrm{norm} 1}(y)=\frac{c}{\exp \left[c y^{b} /\left(1-h y^{b}\right)\right]-1} \Theta\left(1-h y^{b}\right) .
$$


This normalization was used in Ref. [17] in order to study the critical behaviour of the three-dimensional $O(N=1)$ scalar model in LPA and in order to investigate the scheme-dependence of RG equations in Quantum Einstein Gravity [20]. Equation (13) has the following limits,

$$
\begin{aligned}
\lim _{c \rightarrow 0, h \rightarrow 1} r_{\mathrm{css}}^{\mathrm{norm} 1} & =\left(\frac{1}{y^{b}}-1\right) \Theta(1-y), \\
\lim _{c \rightarrow 0, h \rightarrow 0} r_{\mathrm{css}}^{\mathrm{norm} 1} & =\frac{1}{y^{b}}, \\
\lim _{c \rightarrow 1, h \rightarrow 0} r_{\mathrm{css}}^{\mathrm{norm} 1} & =\frac{1}{\exp \left[y^{b}\right]-1} .
\end{aligned}
$$

These relations identify the "Litim limit" $c \rightarrow 0, h \rightarrow 1$, the "power law limit" $c \rightarrow 0, h \rightarrow 0$, and the "exponential limit" $c \rightarrow 1, h \rightarrow 0$ of the propagator. The normalization (13) corresponds to one of the simplest possible choices for $y_{0}$, and a CSS regulator of this kind (we refer to it as the CSS regulator with linear norm) recovers the optimized power-law (with $b=2$ ) and optimized Litim (with $b=1$ ) regulators but it cannot reproduce the exponential regulator given in Eq. (7) with optimal parameters $\left[c_{2}=\ln (2)\right]$.

However, one can choose a different normalization (let us refer to this as the CSS regulator with exponential norm),

$$
r_{\mathrm{css}}^{\mathrm{norm} 2}(y)=\frac{\exp [\ln (2) c]-1}{\exp \left[\frac{\ln (2) c y^{b}}{1-h y^{b}}\right]-1} \Theta\left(1-h y^{b}\right)=\frac{2^{c}-1}{2^{\frac{c y^{b}}{1-h y^{b}}}-1} \Theta\left(1-h y^{b}\right),
$$

where the limits are

$$
\begin{aligned}
\lim _{c \rightarrow 0, h \rightarrow 1} r_{\mathrm{css}}^{\mathrm{norm} 2} & =\left(\frac{1}{y^{b}}-1\right) \Theta(1-y), \\
\lim _{c \rightarrow 0, h \rightarrow 0} r_{\mathrm{css}}^{\mathrm{norm} 2} & =\frac{1}{y^{b}}, \\
\lim _{c \rightarrow 1, h \rightarrow 0} r_{\mathrm{css}}^{\mathrm{norm} 2} & =\frac{1}{\exp \left[\ln (2) y^{b}\right]-1} .
\end{aligned}
$$

The advantage of this type of normalization is that the form (15) reproduces all the major types of regulators with optimal parameters including the exponential one. This type of CSS regulator has also been studied in Ref. [16] beyond the LPA.

Here, we consider both forms of the CSS regulator (13) and (15). The goal is to find the optimal choice of the parameters in LPA by using the PMS method in the framework of two different models in two different dimensions.

\section{$2.3 \mathrm{QED}_{2}$}

One of the advantages of considering two-dimensional field theories is the existence of bosonization transformations. Hence, gauge and fermionic models can be equivalently rewritten $[14,21]$ in terms of scalar fields, and in many cases, one can map the theories onto sine-Gordon type models [22]. For example, we may consider $\mathrm{QED}_{2}$ with a massive Dirac fermion which is also called the massive Schwinger model

$$
\mathcal{L}_{\mathrm{QED}_{2}}=\bar{\psi}\left(i \gamma^{\mu} \partial_{\mu}-m-e \gamma^{\mu} A_{\mu}\right) \psi-\frac{1}{4} F_{\mu \nu} F^{\mu \nu},
$$

where $F_{\mu \nu}=\partial_{\mu} A_{\nu}-\partial_{\nu} A_{\mu}$ is the field-strength tensor $(\mu, \nu=0,1)$. This model can be mapped onto an equivalent Bose form, namely, the specific form of the massive sine-Gordon (MSG) model [14] whose Lagrangian density is written as

$$
\mathcal{L}_{\mathrm{MSG}}=\frac{1}{2}\left(\partial_{\mu} \varphi\right)^{2}+\frac{1}{2} M^{2} \varphi^{2}+u \cos (\beta \varphi) .
$$

Here, $\beta^{2}=4 \pi, M^{2}=e^{2} / \pi, u=e m \exp \left(\gamma_{E}\right) /\left(2 \pi^{3 / 2}\right), \gamma=0.57721 \ldots$ is the Euler-Mascheroni constant, and the vacuum angle parameter (a byproduct of the bosonization) is conveniently chosen as $\theta= \pm \pi$ for $u>0$ and $\theta=0$ 
for $u<0$ (see Ref. [14]). The MSG model has two phases [14]. The Ising-type phase transition is controlled by the dimensionless quantity $u / M^{2}$ related to the critical ratio $(m / e)_{c}$ of $\mathrm{QED}_{2}$ which separates the confining and the halfasymptotic phases of the fermionic model.

The critical ratio $0.13<(\mathrm{m} / \mathrm{e})_{c}<0.33$ has been calculated by the density matrix RG method for the fermionic model [23], implying that $0.156<\left[u / M^{2}\right]_{c}<0.168$. In the framework of functional RG, the preferred result in LPA for the critical ratio reads $\left[u / M^{2}\right]_{c}=2 /(4 \pi) \approx 0.15915$. This result can be determined by analytic considerations based on the infrared (IR) limit of the propagator, $\lim _{k \rightarrow 0}\left(k^{2}+V_{k}^{\prime \prime}(\varphi)\right)=0$, where $V_{k}(\varphi)$ is the blocked scaling potential which contains the mass term and all the higher harmonics generated by RG transformations [14]. However, if one considers a single Fourier-mode approximation [where $V_{k}(\varphi)$ contains the mass term and only a single cosine], then the analytic result based on the IR behavior of the propagator $[24,14]$ gives

$$
\chi_{c}=\left[\frac{u}{M^{2}}\right]_{c}=\frac{1}{(4 \pi)} \approx 0.07957 .
$$

In this case, the optimized regulator (9) with $b=1$ and $c=0.01$ leads to a ratio $\left[u / M^{2}\right]_{c}=0.07964$ closer to the analytic one [14] but other regulators such as the power-law type regulator with $b=1$ run into a singularity and stop at some finite momentum scale, rendering the determination of the critical ratio [14] impossible. Therefore, the use of the single Fourier mode approximation provides us with a tool to consider the convergence properties of the RG equations and to optimize the regulator functions. Here, we use the PMS method in order to determine the optimized parameters of the CSS regulator with various normalizations [see Eqs. (13) and (15)] and compare the ratio given by the CSS regulator with these optimized parameters to the analytic result (19) which represents the optimum available ratio in the case of a single-Fourier-mode approximation.

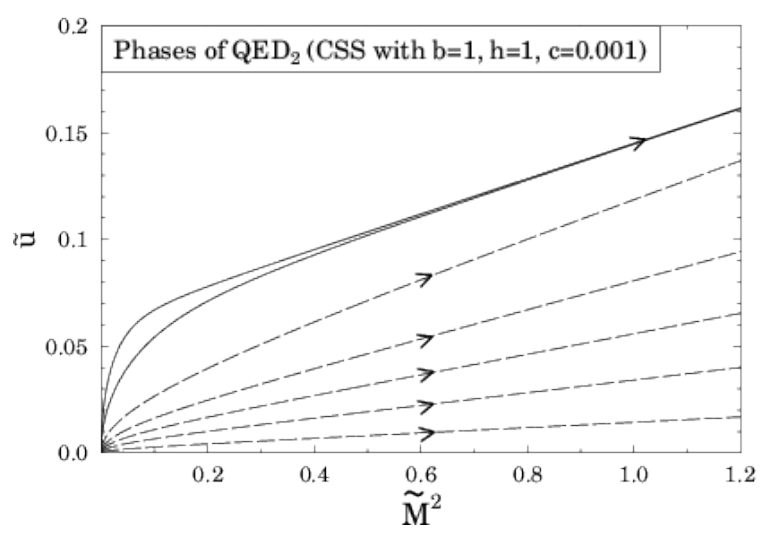

Figure 1: Phase diagram of the MSG model for $\beta^{2}=4 \pi$ is obtained by the usage of the CSS regulator (13) with $b=1, h=1$ and $c=0.001$. RG trajectories of the broken symmetric phase (full lines) merge into a single one and the critical ratio of the model is determined by its slope in the IR limit. The arrows indicate the direction of the flow.

\section{Results obtained by the RG study of QED $_{2}$}

\subsection{Orientation}

We consider the RG study of the bosonized $\mathrm{QED}_{2}$ described by the Lagrangian (18). We use two types of approximations, (i) RG equations are obtained within the LPA [see Eq. (4)], (ii) a single-mode Fourier approximation. In the latter case, the dimensionless blocked potential $\left(\tilde{V}_{k}=k^{-2} V_{k}\right)$ with a single Fourier mode is used in the form

$$
\tilde{V}_{k}=\frac{1}{2} \tilde{M}_{k}^{2} \varphi^{2}+\tilde{u}_{k} \cos (\beta \varphi),
$$




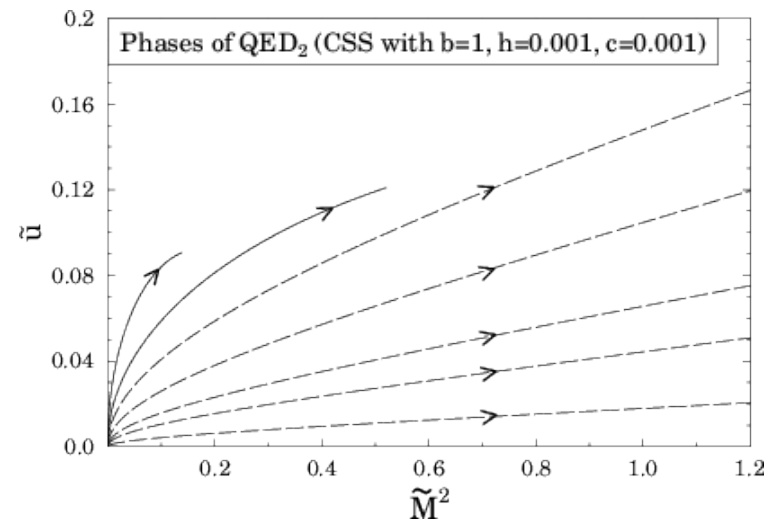

Figure 2: Phase diagram of the MSG model for $\beta^{2}=4 \pi$ is obtained by the usage of the CSS regulator (13) with $b=1, h=0.001$ and $c=0.001$, approximating the power-law limit. RG trajectories of the broken symmetric phase (full lines) stop at some finite momentum scale $k_{\mathrm{f}}$ before they merge into a single one, and the determination of the critical ratio is not possible.

with $\beta^{2}=4 \pi$, and the tilde superscript denotes the dimensionless couplings, $\tilde{M}^{2}=k^{-2} M^{2}$ and $\tilde{u}_{k}=k^{-2} u_{k}$. This is inserted into the dimensionless form of Eq. (4) in $d=2$ which reads as

$$
\left(2+k \partial_{k}\right) \tilde{V}_{k}(\varphi)=-\int_{0}^{\infty} \frac{\mathrm{d} y}{4 \pi} \frac{d r}{d y} \frac{y^{2}}{(1+r) y+\tilde{V}_{k}^{\prime \prime}(\varphi)} .
$$

We emphasize that no further approximations such as the amplitude expansion discussed in Sec. 2.1 are used, here.

The phase structure of the single-frequency MSG model (18), as given in Fig. 1, can be obtained from the functional RG equation (21) using the CSS regulator in "normalization 1 " (13) with $b=1, h=1$ and $c=0.001 \approx 0$. If the CSS regulator is normalized according to Eq. (15) ("normalization 2"), then almost identical results are obtained because both normalizations (13) and (15), tend to Litim's optimized form in the limit $c \rightarrow 0$ (this was also demonstrated in Ref. [13]). All numerical calculations reported here have been obtained via a combination of $\mathrm{C}++$ codes and computer algebra software [25].

The RG trajectories represented by full lines in Fig. 1 correspond to the broken phase of the single-frequency MSG model (18) where the reflection symmetry $\left(Z_{2}\right)$ is broken spontaneously. These RG trajectories merge into a single one in the IR limit and its slope defines the critical ratio. Thus, in the broken phase $\tilde{u}_{k}$ is a linear function of $\tilde{M}_{k}^{2}$ in the IR limit,

$$
\tilde{u}_{k}=a \tilde{M}_{k}^{2}+b .
$$

Both the ratio of the dimensionful coupling to the mass term, as well as the dimensionless equivalent tend to the constant $\left[\tilde{u}_{k \rightarrow 0} / \tilde{M}_{k \rightarrow 0}^{2}\right]=\left[u_{k \rightarrow 0} / M_{k \rightarrow 0}^{2}\right]=a$ (since $\tilde{u}_{k}$ and $\tilde{M}_{k}^{2}$ are increasing in the IR limit, their ratio tends to $a$ and the constant $b$ term can be neglected), and the slope is independent of the initial conditions (in the symmetric phase, the linear functional form (22) holds but the slope depends on the initial values).

There is strong numerical evidence, obtained in this work, for a scheme-independent slope $a=1 /(4 \pi)$, within the single Fourier-mode approximation. However, the convergence properties of the RG equation depend on the regulator chosen. The RG evolution stops at some finite scale $k_{\mathrm{f}} \neq 0$ and the ratio $\left[u_{k \rightarrow k_{\mathrm{f}}} / M_{k \rightarrow k_{\mathrm{f}}}^{2}\right]$ becomes scheme-dependent, where the optimization can be performed. This strategy is used to select the optimized regulator according to its convergence properties. As an example for a special situation, one has to mention the power-law limit $(h \rightarrow 0, c \rightarrow 0)$ of the CSS regulators given in Eqs. (14b) and (16b). The power-law regulator has poor convergence properties for $b=1$, and thus the RG trajectories of the broken phase cannot merge into a single one and the determination of the critical ratio is not possible at all. This is shown in Fig. 2 for the CSS regulator (14b) with $b=1, h=0.001 \approx 0$ and $c=0.001 \approx 0$, approximating the power-law limit. The same conclusion can be obtained on the basis of Eq. (16b). 

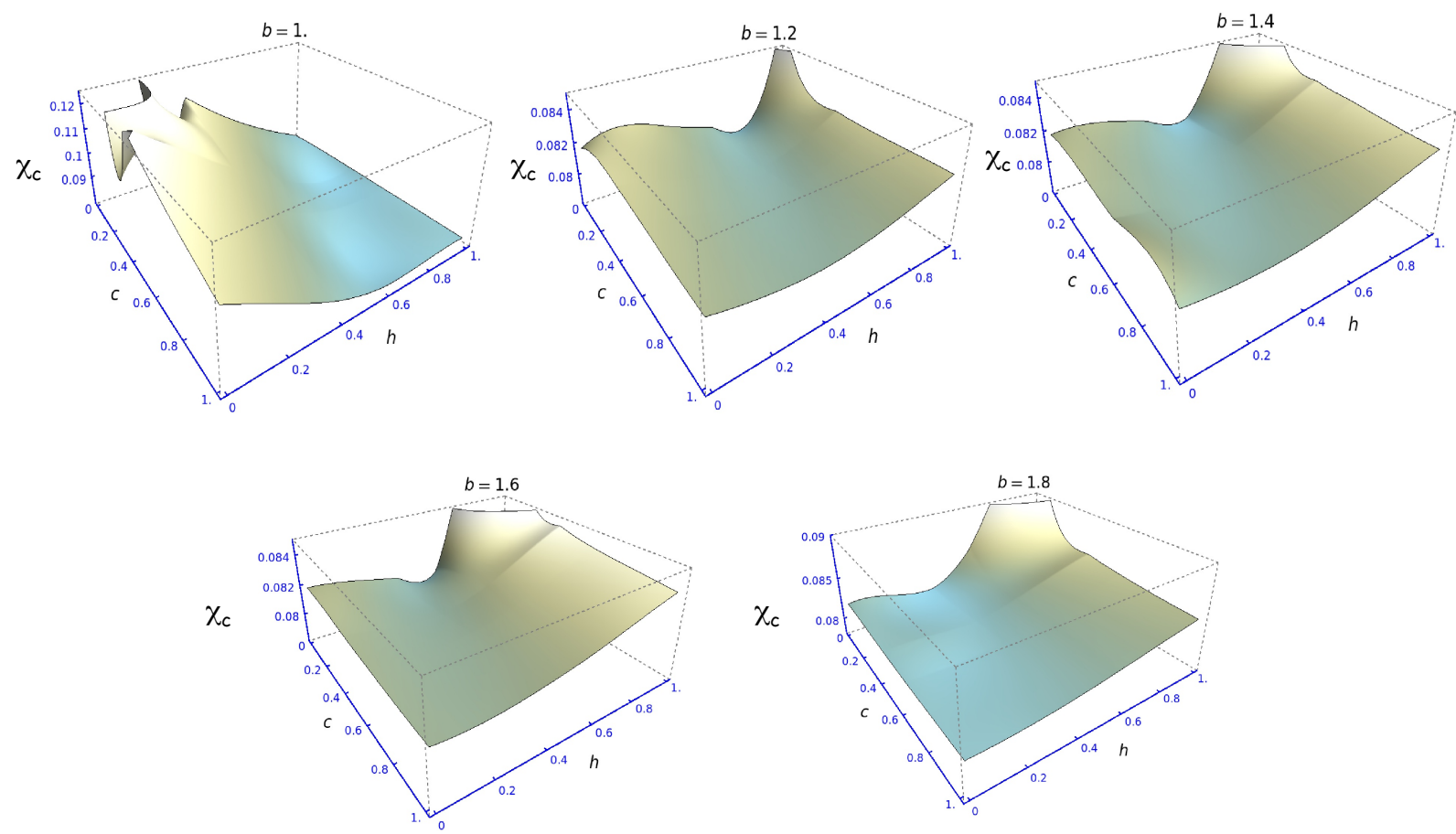

Figure 3: The critical ratio $\chi_{c}$, defined according to Eq. (19), of bosonized $\mathrm{QED}_{2}$, obtained with the CSS regulator in the "linear" normalization (13) for various parameters. Lower ratios indicate better regulators. The optimum result is obtained for $b=1, c=0.001$ and $h=1$ [ "back corner" of Fig. (a)] which is numerically close to the Litim limit of the CSS, according to Eq. (14a). The critical ratio $\chi_{c}$ is on the ordinate axis.

\section{2 $\mathrm{QED}_{2}$ and CSS regulator with linear norm}

We first attempt to find the optimal set of parameters $b, h, c$ for the CSS regulator with the linear norm (13). In Fig. 3, the critical ratio $\left[u / M^{2}\right]_{c}=\tilde{u}_{k \rightarrow k_{f}} / \tilde{M}_{k \rightarrow k_{f}}^{2}=u_{k \rightarrow k_{f}} / M_{k \rightarrow k_{f}}^{2}$ obtained by the CSS with linear norm is shown as a function of $b, h$ and $c$. The lower the ratio the better the regulator is. We obtain results closest to the analytical value (19) for the parameters $b=1, c=0.001$ and $h=1$, which are very close to the Litim limit of the CSS. Let us apply the PMS strategy in order to determine the optimized set of parameters of the CSS regulator which is chosen by finding the extremum (in this particular case the minimum) of a given physical parameter (for $\mathrm{QED}_{2}$ the critical ratio $\left[u / M^{2}\right]_{c}$ ) obtained by the RG method. Thus, near the optimized parameters, the results obtained by the RG study of the model has a minimal sensitivity on the change of parameters. For example, one finds a plateau in Fig. 3 for every subgraph (where the parameter $b$ is fixed) which defines the optimized parameters. For $b=1$, the optimized value as a function of the two remaining arguments is the Litim limit $c \rightarrow 0, h=1$. For $b \neq 1$, the optimized parameters are found in the limit $c \rightarrow 0$, but at $h \neq 1$, still leading to less effective global optimization of the critical ratio.

This can be seen in Fig. 4, where we plot the minimum values for the critical ratio obtained at every subgraph of Fig. 3 in terms of the remaining parameters $c$ and $h$, for "fixed" value of $b$. The dependence of each minimum, found as a function of $c$ and $h$, can thus be read off as a function of $b$, demonstrating that the limit $b \rightarrow 1, c \rightarrow 0$ and $h=1$ leads to the globally most effective optimization. 


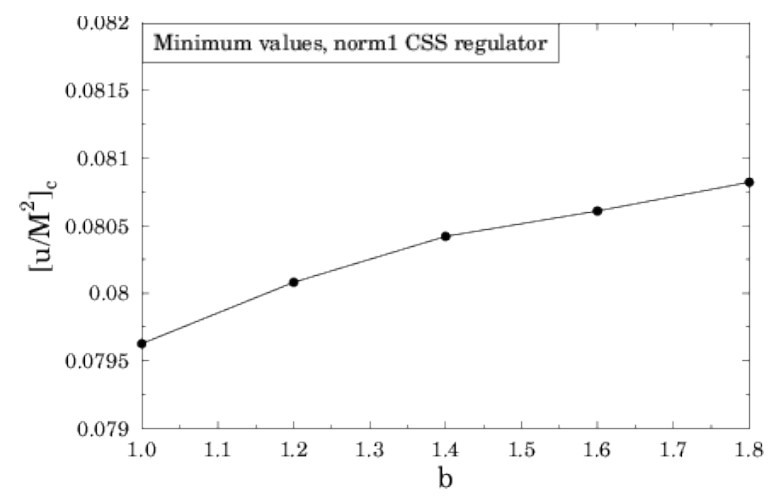

Figure 4: Minimum values (with respect to $c$ and $h$ for a fixed $b$ ) of the critical ratio obtained by the CSS regulator with linear norm are shown as a function of the parameter $b$. It demonstrates that the limit $b \rightarrow 1, c \rightarrow 0$ and $h=1$ gives the numerically most favorable results.

\section{3 $\mathrm{QED}_{2}$ and CSS regulator with exponential norm}

Let us repeat the calculation done in the previous subsection with the CSS, this time employing the exponential norm (15) instead of the linear normalization (13). The goal is the same, i.e., to find the optimal set of parameters $b, h, c$. In Fig. 5 , the critical ratio obtained by the CSS with exponential norm is shown as a function of $b, h$ and $c$. In analogy to the previous analysis for linear norm, the optimum result, i.e., the one closest to the analytic formula (19), is obtained for $b=1, c=0.001$ and $h=1$. This, again, is the Litim limit of the CSS regulator. In Fig. 6 , we plot the minimum values for the critical ratio obtained at every subgraph of Fig. 5, i.e., keeping $b$ fixed, and thus the dependence of each minimum on the parameter $b$ can be read off, confirming once more the nature of the optimized propagator. Again, this demonstrates that Litim's limit $(b \rightarrow 1, c \rightarrow 0$ and $h=1)$ leads to the optimum results. The PMS strategy gives us the same optimal choice for the parameters of the CSS regulator, the limit $b \rightarrow 1, c \rightarrow 0$ and $h=1$, independent of the normalization of the CSS regulator. In order to gain more accurate information on the optimal choice of the parameters for the CSS regulators (13) and (15), we now consider the $O(N=1)$ symmetric scalar field theory in $d=3$ dimensions (which constitues a "textbook example").

\section{Three-dimensional $O(1)$ model}

\subsection{Orientation and previous studies}

A further textbook example for the optimization of the parameters entering the propagator is given by the $O(1)$ model in $d=3$ dimensions around the Wilson-Fisher (WF) fixed point and the corresponding critical exponent $\nu$ (which describes the critical behaviour of the correlation length). Similar to the study of the bosonized $\mathrm{QED}_{2}$ with a single Fourier mode, the $O(1)$ model is investigated here using a drastic truncation (as drastic as possible), i.e., the dimensionless potential is defined as

$$
\tilde{V}=\frac{1}{2} \tilde{g}_{1} \tilde{\varphi}^{2}+\frac{1}{4 !} \tilde{g}_{2} \tilde{\varphi}^{4},
$$

with the dimensionless couplings $\tilde{g}_{1}$ and $\tilde{g}_{2}$. With this truncation, the RG flow equations obtained for the couplings read as [17],

$$
\begin{aligned}
& \dot{\tilde{g}}_{1}=-2 \tilde{g}_{1}+\tilde{g}_{2} \bar{\Phi}_{3 / 2}^{2}\left(\tilde{g}_{1}\right), \\
& \dot{\tilde{g}}_{2}=-\tilde{g}_{2}+6 \tilde{g}_{2}^{2} \bar{\Phi}_{3 / 2}^{3}\left(\tilde{g}_{1}\right),
\end{aligned}
$$



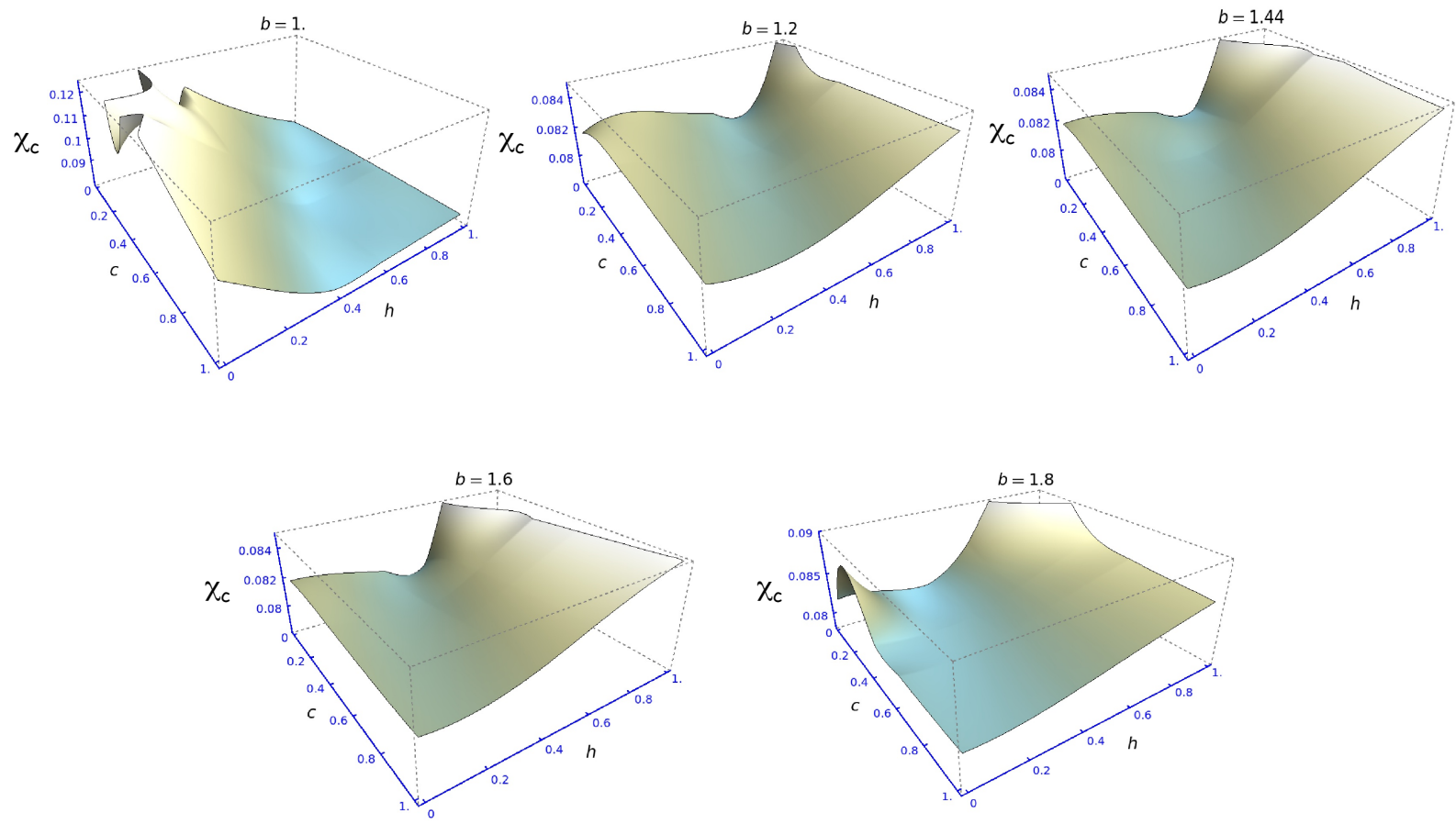

Figure 5: We plot the critical ratio $\chi_{c}$ of bosonized $\mathrm{QED}_{2}$, obtained by various parameters of the CSS regulator with exponential norm (15). The critical ratio $\chi_{c}$ is on the ordinate axis. Lower critical ratios indicate better regulators, with the optimum results being obtained for $b=1, c=0.001$ and $h=1$ (Litim limit of the CSS). The geometry of the plots is similar to those recorded in Fig. 3 but numerical differences are observed. For larger values in the range $b \approx 1.8$, an additional local maximum develops as a function of $c$, along the $(h=0)$-manifold.

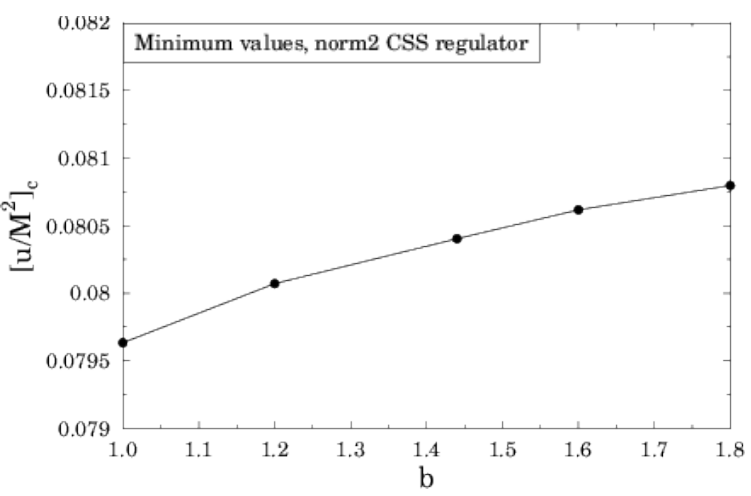

Figure 6: Minimum values (with respect to $c$ and $h$ for fixed $b$ ) of the critical ratio obtained by the CSS regulator with exponential norm are plotted for various values of the parameter $b$. Again, the most favorable results are obtained in the limit $b \rightarrow 1, c \rightarrow 0$ and $h \rightarrow 1$. Notably, the optimum critical ratio $\left[u / M^{2}\right]_{c}$ obtained for $b \rightarrow 1$ is lower than the "optimum" value (with respect to a variation of $c$ and $h$ ) obtained for $b=1.44$.

where the threshold function is introduced according to

$$
\bar{\Phi}_{n}^{p}(\omega)=\frac{1}{(4 \pi)^{n} \Gamma(n)} \int_{0}^{\infty} \mathrm{d} y \frac{y^{n+1} r^{\prime}}{(y(1+r)+\omega)^{p}} .
$$



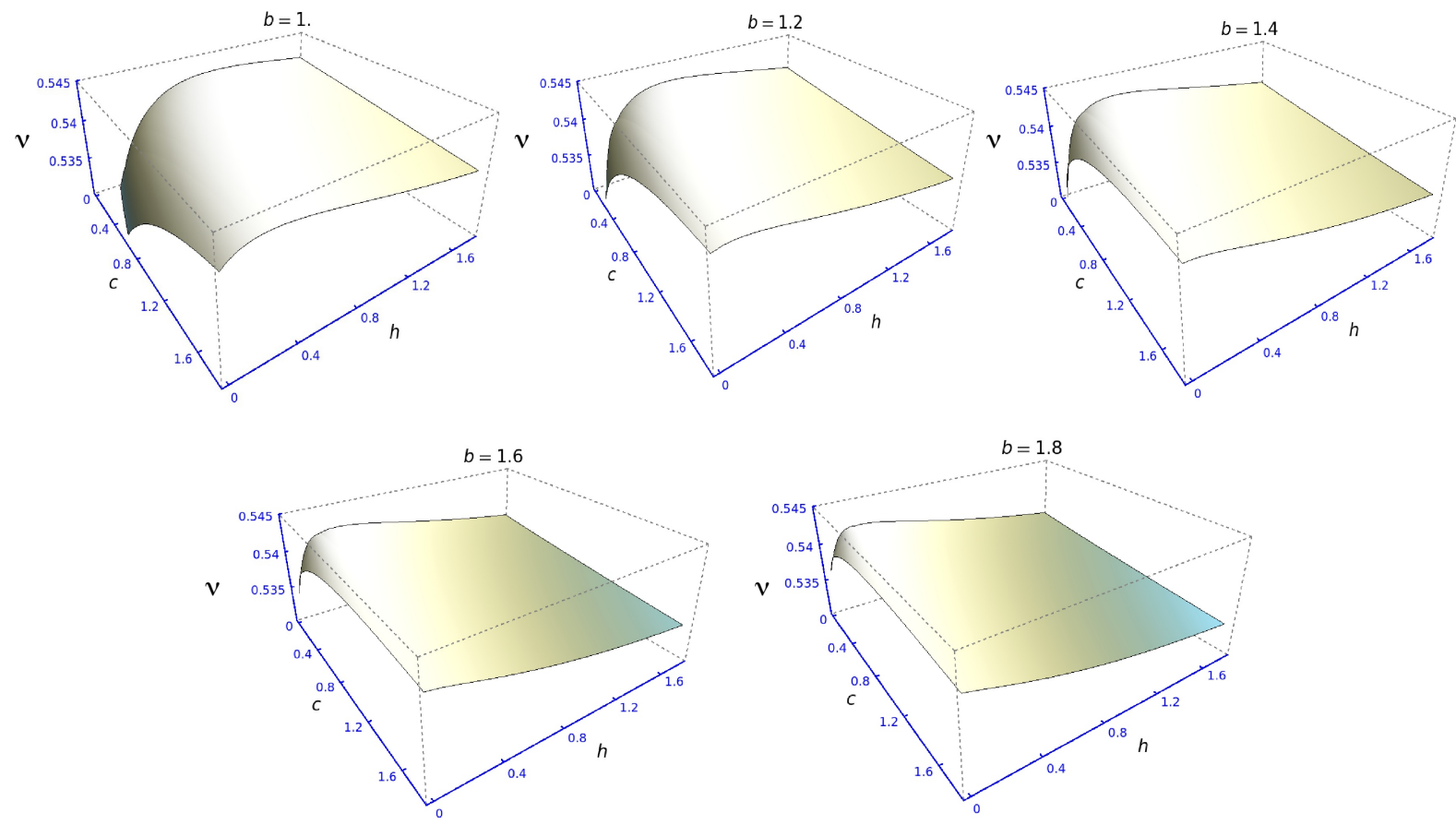

Figure 7: The critical exponent $\nu$ of the three-dimensional $O(1)$ model with two couplings, as given by the potential of Eq. (23), is obtained by various parameters of the CSS regulator with exponential norm (15). Higher exponents indicate better regulators. The critical exponent $\nu$ is plotted on the ordinate axis.

The flow equations obviously are regulator-dependent, and the exponent $\nu$ calculated near the WF fixed point is regulatordependent too. The threshold function has been calculated for all major types of regulators in the literature, see e.g. $[6,7,8,9]$. Among these regulators, the Litim regulator is known to give us the "best" critical exponent (closest to the "exact" value). For two couplings, the result for $\nu$ obtained using the Litim form of the regulator reads as $\nu \approx 0.54277$ $[17]$.

The CSS regulator with the linear norm (13) has been studied in Ref. [17] using the RG evolution for 2 and 4 couplings, where it has been confirmed that the optimized parameters of the CSS with linear norm are those of the Litim limit., supporting the observations made in Sec. 3. However, the CSS with exponential norm has not yet been studied in the framework of the $O(1)$ model, so we perform a detailed analysis of the CSS regulator in the normalization (15) looking for the optimal set of parameters.

\subsection{Three-dimensional $O(1)$ model and CSS regulator with exponential norm}

In this subsection, we perform the RG study of the $O(1)$ model (23) by the CSS regulator with exponential norm (15). Our goal here is the same as it was in the case of $\mathrm{QED}_{2}$, namely, we are looking for the optimal set of parameters $(b, h, c)$. Here, the critical exponent $\nu$ of the $O(1)$ model (with 2 couplings) is determined and one finds the optimized parameters at which $\nu$ is maximal. In Fig. 7 , we plot the critical exponent $\nu$ obtained by the CSS regulator with exponential norm as a function of $b, h$ and $c$.

We apply the PMS strategy. Near the optimized set of parameters, the results obtained for $\nu$ by the RG study of the 


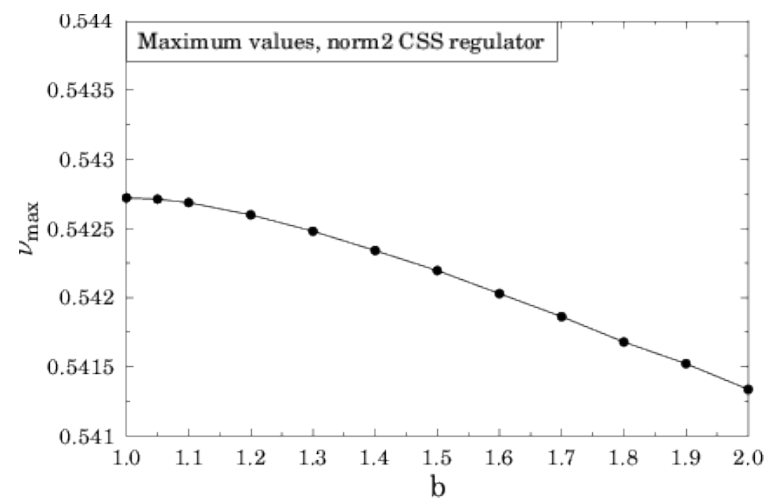

Figure 8: The maximum values of the critical exponent $\nu$ obtained by the CSS regulator with exponential norm (15) for various values of the parameter $b$ is shown. Using the PMS method, one finds that the Litim limit $b \rightarrow 1$ leads to the optimum result.

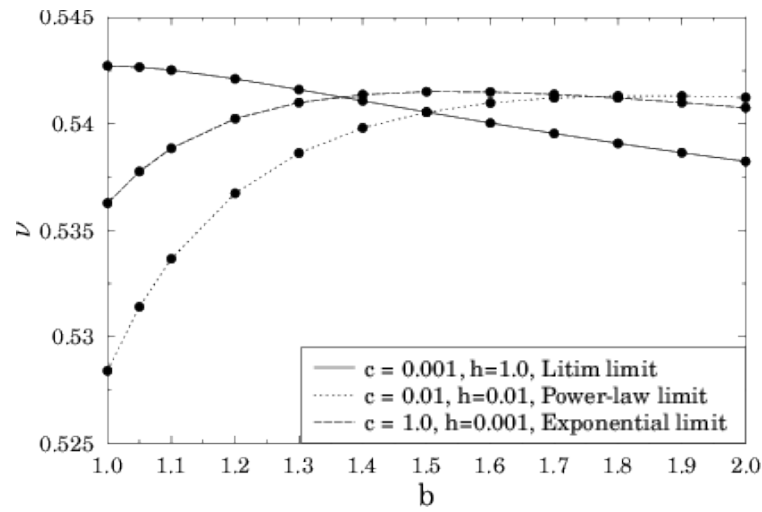

Figure 9: The $b$-dependence of the critical exponent $\nu$ obtained by the CSS regulator with exponential norm (15) in various limits is shown.

model have minimal sensitivity on small perturbations. Around these optimized parameters, one finds plateaus in Fig. 7 for every subgraph. If we identify $\nu_{\max }$ as the maximum of $\nu$ with respect to $c$ and $h$ of every subgraph of Fig. 7, and we plot it against the parameter $b$, see Fig. 8, one can read off the absolute maxima using the PMS principle. According to the PMS method, the optimum result is obtained for $b=1, c=0.001$ and $h=1$ which is the (numerically close to) Litim limit of the CSS regulator. We recall that both normalizations (13) and (15) converge to the Litim limit for $c \rightarrow 0$, and $h=1$.

Another perspective on the problem is obtained when we consider the CSS regulator for fixed values of the parameters $c$ and $h$, and investigate the critical exponent $\nu$ as a function of $b$. The results obtained by the CSS regulator regulator with exponential norm (15) in the Litim, power-law and exponential limits are plotted in Fig. 9. In the Litim limit of the CSS, the optimum results obtained by the PMS strategy are obtained for $b=1$ (which is the generally accepted value in the literature). In the power-law limit of the CSS, one finds $b \approx 2$. Based on the field-amplitude expansion, it is known that the optimum choice for the power-law regulator is $b=2$. In the exponential limit of the CSS (15), the most favorable results were obtained for $b \approx 1.5$ (consistent with a visual inspection of Fig. 9), while it is known that, based on the field-amplitude expansion, the optimum choice for the exponential regulator is $b=1.44$. These latter observations are important in order to ensure the consistency of our calculations with known results, for specific, fixed values of the regulator parameters. 


\section{Summary}

In this paper, we have discussed the optimization of the regulator-dependence of functional RG equations in LPA, for the bosonized $\mathrm{QED}_{2}$ and the three-dimensional $O(1)$ scalar theory. The optimization has been done in the framework of the PMS method. It has been known [9, 7, 8] that Litim's regulator leads to the fastest convergence of the field amplitude expansion. This regulator is also known as the optimum regulator in LPA, which yields critical exponents of the $O(N)$ model very close to the "exact" ones. The recently introduced CSS regulator [13], which has a very general functional form [see Eq. (11)], has not yet been subjected to a thorough optimization analysis. The CSS regulator reduces to all major type of regulator functions in appropriate limits [see Eqs. (14) and (16)]. Due to its versatile functional form, the CSS regulator represents an excellent playground for the PMS method allows to systematically investigate the optimization of the regulator within a rather wide class of regulator functions. The CSS regulator has been employed here with two different normalizations (13) and (15) ("linear" and "exponential" norm). In order to numerically study the convergence properties of RG equations with various regulators, we have used a truncation of the field expansion for both models, i.e., a single Fourier mode approximation for the bosonized $\mathrm{QED}_{2}$ and a single polynomial approximation for the $O(1)$ model in three dimensions (i.e. the inclusion of the $\varphi^{4}$ term in the blocked action but no higher polynomials). Our results can be summarized as follows.

(i) Litim's form of the CSS regulator has been found to be the optimum choice in both cases. In particular, we have been unable to find a set of parameters of the general CSS regulator, which would otherwise lead to numerically more favorable results as compared to those obtained with Litim's form. This statements holds for both $\mathrm{QED}_{2}$ as well as the three-dimensional $O(1)$ model.

(ii) The PMS method can be applied to the RG analysis of the CSS very effectively. In particular, it allows us to search for the numerically optimal set of parameters within a three-dimensional space of parameters $b, h$ and $c$ describing the general form of the CSS regulator (11). We confirm that Litim's form of the regulator leads to the numerically most favorable results within the LPA. Such a general investigation based on the PMS method has not been recorded in the literature to the best of our knowledge. Regarding possible future investigations, we would like to expand that in principle, the combination of the CSS regulator and the PMS was found to be a very powerful method which can be applied in any dimension, for any type of models, in any order of the gradient expansion.

(iii) Various known results were recovered by using the CSS regulator in specific limits. For example, in the exponential limit of the CSS, for the three-dimensional $O(1)$-model, the optimum results for the critical exponent $\nu$ were obtained for $b \approx 1.5$, which compares well with the field-amplitude expansion, where the known optimized value for the exponential regulator is $b=1.44$.

Finally, let us note that the CSS regulator is infinitely differentiable for any nonzero values of $c$ and $h$, while in the exact limit $c \rightarrow 0$ advocated by Litim, a kink develops at $y=1$. However, for any manifestly nonzero value of $c$, say, $c=0.001$, it can be applied to higher order terms of the gradient expansion in any order, unlike Litim's original regulator which is non-differentiable. This would indicate that the form of an "optimized CSS regulator" with a small, but nonzero value of $c$, remains both compatible with the gradient expansion while reproducing the optimum values for the critical ratio $\chi_{c}$ and the critical exponent $\nu$ in appropriate limits. A nonvanishing, small but finite value of the $c$ parameter regularizes the kink of the regulator functions (at the end point of the compact support of the regulator). The essential singularity of the exponential function at infinity is crucial in the regularization process. Let us study the regulator (13) for $b=1$,

$$
r_{\mathrm{css}}^{\mathrm{norm} 1}(y)=\frac{c}{\exp [c y /(1-h y)]-1} \Theta(1-h y),
$$

with a special emphasis on the limit $c \rightarrow 0$. We first observe that, globally, for $c \rightarrow 0$, the regulator retains a finite limit, because the small factor $c$ in the numerator cancels against the same factor from the denominator. One might ask if divergences could occur for small values of $c$ when higher-order derivative terms are considered. Preliminary results, based on example calculations, indicate that this is not the case. Typical integrals which occur in calculations beyond the LPA, for the sine-Gordon models in one and two dimensions, have been given in Eqs. (8) and (9) of Ref. [16]. The distance $D$ between the non-trivial IR fixed point and the saddle point of the RG flow, for the mentioned sine-Gordon models, has been given in Eq. (10) of Ref. [16]. Preliminary numerical numerical suggests that no additional divergences occur 
for small values of $c$, even if derivatives of the regulator function are taken according to Eqs. (8) and (9) of Ref. [16]. This is consistent with the nonperturbative character of the CSS regulator near the end point of the compact support interval, due to the essential singularity of the exponential function at infinity. (Further details will be discussed elsewhere.) Thus, there is hope that in future studies on related problems, the CSS regulator, with parameters close to the Litim limit, might be very useful in advances beyond the LPA, and may recommend a regularized, and numerically optimized form of the regulator. Identifying the optimum form of the regulator function is important in order to improve the general understanding of the scheme dependence of the functional renormalization group.

\section{Acknowledgements}

This work was partially supported by the European Union and the European Social Fund through project Supercomputer, the national virtual lab (grant no.: TAMOP-4.2.2.C-11/1/KONV-2012-0010). U.D.J. acknowledges support from the National Science Foundation (Grant PHY-1068547) and from the National Institute of Standards and Technology (precision measurement grant).

\section{References}

[1] F. J. Wegner, A. Houghton, Phys. Rev. A. 8, 401 (1973); J. Polchinski, Nucl. Phys B 231, 269 (1984).

[2] C. Wetterich, Nucl. Phys. B 352, 529 (1991); ibid, Phys. Lett. B301, 90 (1993); J. Berges, N. Tetradis, C. Wetterich, Phys. Rept. 363, 223 (2002).

[3] T. R. Morris, Int. J. Mod. Phys. A 9, 2411 (1994);

[4] J. Alexandre, J. Polonyi, Annals Phys. 288, 37 (2001); J. Alexandre, J. Polonyi, K. Sailer, Phys. Lett. B 531, 316 (2002); J. Polonyi, Central Eur. J. Phys.1, 1 (2004).

[5] D. F. Litim and J. M. Pawlowski, Phys. Rev. D 66, 025030 (2002).

[6] R. D. Ball, P. E. Haagensen, J. I. Latorre and E. Moreno, Phys. Lett. B 347, 80 (1995); D. F. Litim, Phys. Lett. B 393, 103 (1997); K. Aoki, K. Morikawa, W. Souma, J. Sumi and H. Terao, Prog. Theor. Phys. 99, 451 (1998); S.B. Liao, J. Polonyi, M. Strickland, Nucl. Phys. B 567, 493 (2000); J. I. Latorre and T. R. Morris, JHEP 0011, 004 (2000); F. Freire and D. F. Litim, Phys. Rev. D 64, 045014 (2001); D. F. Litim, JHEP 0507, 005 (2005); C. Bervillier, B. Boisseau, H. Giacomini, Nucl. Phys. B 789, 525 (2008); C. Bervillier, B. Boisseau, H. Giacomini, Nucl. Phys. B 801, 296 (2008); C. S. Fischer, A. Maas, J. M. Pawlowski, Ann. Phys. 324, 2408 (2009).

[7] D. F. Litim, Phys. Lett. B 486, 92 (2000); ibid, Phys. Rev. D 64, 105007 (2001); ibid, JHEP 0111, 059 (2001).

[8] J. M. Pawlowski, Ann. Phys. (N.Y.) 322, 2831 (2007).

[9] D. F. Litim, Nucl.Phys. B 631, 128 (2002).

[10] T. R. Morris, JHEP 0507, 027 (2005).

[11] O. J. Rosten, Phys. Rept. 511, 177 (2012).

[12] L. Canet, B. Delamotte, D. Mouhanna and J. Vidal, Phys. Rev. D 67, 065004 (2003); ibid, Phys. Rev. B 68064421 (2003); L. Canet, Phys.Rev. B 71012418 (2005).

[13] I. Nándori, JHEP 1304, 150 (2013).

[14] I. Nándori, Phys. Rev. D 84, 065024 (2011). 
[15] P. Salamon, T. Vertse, Phys. Rev. C 77, 037302 (2008); P. Salamon, A. T. Kruppa, T. Vertse, Phys. Rev. C 81, 064322 (2010).

[16] I. Nándori, I. G. Márián, V. Bacsó, Phys. Rev. D 89, 047701 (2014).

[17] S. Nagy, B. Fazekas, L. Juhasz, K. Sailer, e-Print: arXiv:1307.0765 [hep-th].

[18] I. Nándori, S. Nagy, K. Sailer, A. Trombettoni, Phys. Rev. D 80, 025008 (2009); ibid, JHEP 1009, 069 (2010).

[19] S. Nagy, K. Sailer, Annals Phys.326, 1839 (2011).

[20] M. Reuter, Phys. Rev. D 57, 971 (1998); M. Reuter, F. Saueressig, New J. Phys. 14. 055022 (2012); D. F. Litim, Phys. Rev. Lett. 92, 201301 (2004); M. Reuter, F. Saueressig, Phys. Rev D 65, 065016 (2002); S. Nagy, J. Krizsan, K. sailer, JHEP 1207102 (2012); S. Nagy, arXiv:1211.4151 [hep-th].

[21] S. Nagy, J. Polonyi, K. Sailer, Phys. Rev. D 70, 105023 (2004); I. Nándori, J. Phys. A: Math. Gen. 39, 8119 (2006); I. Nándori, Phys. Lett. B 662, 302 (2008); S. Nagy, Phys. Rev. D 79, 045004 (2009); J. Kovács, S. Nagy, I. Nándori, K. Sailer, JHEP 1101, 126 (2011).

[22] S. R. Coleman Phys. Rev. D 11, 2088 (1975); I. Nándori, J. Polonyi, K. Sailer, Phys. Rev. D 63, 045022 (2001); S. Nagy, K. Sailer, J. Polonyi, J. Phys. A 39, 8105 (2006); S. Nagy, I. Nándori, J. Polonyi, K. Sailer, Phys. Lett. B 647, 152 (2007); V. Pangon, S. Nagy, J. Polonyi, K. Sailer, Phys. Lett. B 694, 89 (2010); I. Nándori, K. Sailer, U. D. Jentschura and G. Soff, J. Phys. G 28, 607 (2002); I. Nándori, K. Sailer, U. D. Jentschura, G. Soff, Phys. Rev. D 69, 025004 (2004); S. Nagy, I. Nándori, J. Polonyi, K. Sailer, Phys. Rev. Lett. 102241603 (2009). J. Alexandre, D. Tanner, Phys. Rev. D 82, 125035 (2010); V. Pangon, Int. J. Mod. Phys. A 227, 1250014 (2012). I. Nándori, arXiv:1108.4643 [hep-th].

[23] T. M. Byrnes, P. Sriganesh, R. J. Bursill and C. J. Hamer, Phys. Rev. D 66, 013002 (2002).

[24] S. Nagy, K. Sailer, arXiv:1012.3007 [hep-th]; S. Nagy, Nucl. Phys. B 864, 226 (2012); S. Nagy, Phys. Rev. D 86, 085020 (2012); V. Pangon, S. Nagy, J. Polonyi, K. Sailer, Int. J. Mod. Phys. A26, 1327 (2011).

[25] S. Wolfram, The Mathematica Book (Cambridge University Press, Cambridge, UK, 1999). 